\title{
Prevalence of Radiologically Isolated Syndrome and White Matter Signal Abnormalities in Healthy Relatives of Patients with Multiple Sclerosis
}

T. Gabelic, D.P. Ramasamy, B. Weinstock-Guttman, J. Hagemeier, C. Kennedy, R. Melia, D. Hojnacki, M. Ramanathan, and R. Zivadinov it $0^{-1}$

\begin{abstract}
BACKGROUND AND PURPOSE: The exact prevalence of WM signal abnormalities in healthy relatives of MS patients and their impact on disease development has not been fully elucidated. The purpose of this study was to compare WM signal abnormality characteristics and the prevalence of radiologically isolated syndrome in healthy control subjects selected randomly from the population with the healthy relatives of patients with MS.
\end{abstract}

MATERIALS AND METHODS: Healthy control subjects $(n=150)$ underwent physical and 3T MR imaging examinations. Healthy control subjects were classified as non-familial healthy control subjects $(n=82)$ if they had no family history of MS or as healthy relatives of patients with MS $(n=68)$ if they had $\geq 1$ relative affected with MS. The presence of radiologically isolated syndrome was evaluated according to the Okuda criteria; dissemination in space on MR imaging and fulfillment of radiologically isolated syndrome criteria were also evaluated according to Swanton criteria.

RESULTS: There was a significantly higher total volume of WM signal abnormality in the healthy relatives of patients with MS compared with the non-familial healthy control subjects $(P=.024$ for signal abnormality $\geq 3 \mathrm{~mm}$ in size and $P=.025$ for all sizes). Periventricular localization and the number of lesions in all groups ( $P=.034$ and $P=.043$ ) were significantly higher in the healthy relatives of patients with MS; $8.8 \%$ of the healthy relatives of patients with $\mathrm{MS}$ and $4.9 \%$ of non-familial healthy control subjects showed $\geq 9 \mathrm{WM}$ signal abnormalities; $2.9 \%$ of subjects in the healthy relatives of patients with MS group and $2.4 \%$ of non-familial healthy control subjects fulfilled radiologically isolated syndrome according to the Okuda criteria, whereas $10.3 \%$ and $3.7 \%$ of subjects fulfilled radiologically isolated syndrome according to the Swanton criteria. In the healthy relatives of patients with MS, smoking was associated with the presence of WM signal abnormalities, whereas obesity was related to the presence of $\geq 9 \mathrm{WM}$ signal abnormalities and to fulfillment of radiologically isolated syndrome according to the Swanton criteria.

CONCLUSIONS: The frequency of WM signal abnormalities and radiologically isolated syndrome is higher in the healthy relatives of patients with multiple sclerosis patients compared with non-familial healthy control subjects.

ABBREVIATIONS: DAWM = dirty-appearing white matter; $\mathrm{DIS}=$ dissemination in space; $\mathrm{HC}=$ healthy control subject; non-fHC = non-familial healthy control subject; $\mathrm{PD}$ = proton attenuation; RIS = radiologically isolated syndrome; $\mathrm{SAV}=$ signal abnormality volume

M ultiple sclerosis is an inflammatory autoimmune demyelinating disorder of the CNS. ${ }^{1}$ Although MS is predominantly a sporadic disease, a genetic predisposition to developing

Received March 5, 2013; accepted after revision April 19

From the Buffalo Neuroimaging Analysis Center (T.G., D.P.R., J.H., C.K., R.M., R.Z.) The Jacobs Neurological Institute (B.W.-G., D.H., R.Z.), Department of Neurology, and Department of Pharmaceutical Sciences (M.R.), State University of New York, Buffalo, New York; and Department of Neurology (T.G.), Referral Centre for Demyelinating Disease of the Central Nervous System, University Hospital Centre Zagreb, Zagreb, Croatia.

Please address correspondence to Robert Zivadinov, MD, PhD, FAAN, Department of Neurology, School of Medicine and Biomedical Sciences, The Jacobs Neurological Institute, 100 High St, Buffalo, NY 14203; e-mail: rzivadinov@bnac.net

- Indicates open access to non-subscribers at www.ajnr.org

http://dx.doi.org/10.3174/ajnr.A3653 familial MS is well accepted. ${ }^{2}$ Although the exact definition of familial MS is not yet established, familial patients with MS are considered to be those with $\geq 1$ first-degree relative affected with $\mathrm{MS},{ }^{2}$ although some authors use a definition that is based on the presence of 2 first-degree relatives. ${ }^{3}$ In a recent meta-analysis, the risk of development of MS was 18.2\% for monozygotic twins of patients with MS, $4.6 \%$ for dizygotic twins, and $2.7 \%$ for siblings. ${ }^{4}$ The risk of development of MS in a first-degree relative of the affected patient is 30 - to 50 -fold higher than in the general population, ${ }^{5}$ whereas second- and third-degree relatives also showed an increased risk for development of MS. ${ }^{6}$

Whether familial or non-familial MS are different forms of the disease is not fully elucidated yet. ${ }^{7}$ Differences in disease progression in familial MS were observed in several studies demonstrating earlier age of onset and increased probability of a progressive 
clinical course. ${ }^{8}$ However, other studies did not show clinical differences among familial and non-familial healthy control subject (non-fMS) forms. ${ }^{2,9}$ Nevertheless, MR imaging studies by use of nonconventional techniques showed MR imaging differences between familial and non-familial MS. ${ }^{10-12}$

In 2009, Okuda et al ${ }^{13}$ introduced the term "radiologically isolated syndrome" (RIS) to describe subjects who show incidental brain MR imaging WM lesions suggestive of MS and who fulfill Barkhof criteria for dissemination in space (DIS) ${ }^{14}$ but have no signs or symptoms of the disease. Overall, the prevalence of RIS is, according to postmortem studies, in a range of $0.06-0.7 \%$, with an age range of 16-70. ${ }^{13,15}$ However, the McDonald 2010 criteria for DIS ${ }^{16}$ substituted Barkhof criteria ${ }^{14}$ with the Swanton criteria for DIS. ${ }^{17}$ Swanton DIS criteria require the presence of $\geq 1$ WM lesion in $\geq 2$ of the brain regions (juxtracortical, periventricular, or infratentorial) or in the spinal cord. ${ }^{17}$ The value of the Swanton criteria for DIS ${ }^{17}$ was not evaluated with respect to the diagnosis of RIS.

Previous MR imaging studies showed that asymptomatic relatives of patients with MS display significant magnetization transfer ratio changes in CNS WM signal abnormality, indicative of MS pathology, ${ }^{3,18}$ though some other studies showed no differences in the magnetization transfer ratio of WM in the siblings of patients with MS. ${ }^{19}$ Studies with the use of conventional brain MR imaging showed WM signal abnormalities consistent with MS in the healthy relatives of patients with $\mathrm{MS},{ }^{20-22}$ with $4 \%$ of nonfamilial healthy control subjects (non-fHC) and 10\% of healthy control subjects with familial MS (HC) fulfilling Barkhof criteria $^{14}$ for DIS. ${ }^{20}$ However, these studies had several limitations including the number of enrolled subjects, strength of the MR imaging field, and use of more conservative criteria for RIS. ${ }^{13}$ As a result, the exact prevalence of RIS and the WM signal abnormalities in asymptomatic MS relatives is not yet fully defined.

The aim of this pilot study was to compare WM signal abnormality characteristics in a large cohort of non-fHCs and healthy relatives of patients with MS by use of 3T MR imaging. We also determined the prevalence of RIS in HC groups, according to both the Barkhof ${ }^{14}$ and Swanton ${ }^{17}$ MR imaging criteria for DIS and investigated association between the presence of vascular risk factors and RIS.

\section{MATERIALS AND METHODS \\ Subjects}

This was a pilot study in which HCs without known CNS pathology or neurologic complaints were recruited from the following volunteer sources: hospital personnel, respondents to a local newspaper advertisement, and relatives of the patients with MS who are followed in our center. The inclusion criteria were: fulfilling the health screen questionnaire requirements containing information regarding medical history (illnesses, surgeries, vascular and environmental risk factors, medications, family history of MS, etc), fulfilling the health screen requirements on physical examination, having $\geq 1$ relative with MS followed in our center (for the healthy relatives of patients with MS), and being able to undergo MR imaging scanning. Exclusion criteria included preexisting medical conditions known to be associated with CNS pathology (eg, neurodegenerative disorder, cerebrovascular disease, cognitive impairment, history of psychiatric disorders, seizures, and trauma, etc). All subjects underwent physical, neurologic, and MR imaging examinations and were assessed with a structured ques- tionnaire administered in person by a trained interviewer unaware of the subjects' healthy control status. ${ }^{23}$ Race/ethnicity was determined according to the US Census Bureau.

Subjects with unknown relatives affected by MS were classified as non-fHC and familial HCs were classified according to the number of family members affected by clinically definitive $\mathrm{MS}^{16}$ by use of previously proposed definitions. ${ }^{2,3}$ In particular, sporadic $\mathrm{HCs}$ had to have at least 1 affected relative (first-, second-, or third-degree), whereas familial HCs had to have $\geq 1$ affected firstdegree relative plus $\geq 1$ affected first-, second-, or third-degree relative. Family members were classified into first-, second-, and third-degree relatives as described previously. ${ }^{10}$ There were 47 subjects in sporadic and 21 subjects in familial HC subgroups. Both subgroups were merged into 1 group composed of healthy relatives of patients with MS.

Subjects were evaluated for diagnostic criteria of RIS, proposed by Okuda et al (Table 1 and Fig 1). ${ }^{13}$ If the subjects fulfilled DIS on MR imaging according to Barkhof criteria ${ }^{14}$ and satisfied Okuda diagnostic criteria for RIS, ${ }^{13}$ they were classified as having RIS-Okuda. We also applied modified Okuda criteria ${ }^{13}$ in which the DIS on MR imaging (section A2 of the Okuda criteria) was evaluated according to DIS Swanton criteria (Table 1 and Fig 1). ${ }^{17}$ If the subjects fulfilled these modified RIS criteria, they were classified as having RIS-Swanton. As required in the Okuda criteria, only lesions $\geq 3 \mathrm{~mm}$ were considered in RIS analysis. ${ }^{13}$

The study was approved by our local Health Sciences Institutional Review Board, and written informed consent was obtained from all subjects.

\section{MR Imaging Acquisition}

All subjects were examined on a 3T Signa Excite HD 12.0 Twin Speed 8-channel scanner (GE Healthcare, Milwaukee, Wisconsin). MR imaging sequences included multi-planar dual fast spinecho proton attenuation (PD) and T2WI as well as FLAIR. Pulse sequence characteristics for $3 \mathrm{~T}$ MR imaging were as follows: all scans were acquired with a $256 \times 256$ matrix and a $25.6-\mathrm{cm}$ FOV for an in-plane resolution of $1 \times 1 \mathrm{~mm}^{2}$ with a phase FOV of $75 \%$ and 1 average. Sequence-specific parameters were as follows: for the PD/T2: 3-mm-thick sections with no gap, TE1/TE2/TR = 12/95/ $3000 \mathrm{~ms}$, echo-train length $=14$, and for the FLAIR scans, $3-\mathrm{mm}$ thick sections with no gap, TE/TI/TR $=120 / 2100 / 8500 \mathrm{~ms}$.

\section{MR Imaging Analysis}

The MR imaging analyses were blinded to the subject demographic and clinical characteristics.

To facilitate combined analysis, each subject's relevant T2/PD images were co-registered to the FLAIR by use of a rigid body ( 6 df) registration. ${ }^{24}$ The FLAIR image was used to outline WM signal abnormalities, whereas the co-registered T2/PD images were used to confirm the presence of WM signal abnormalities. Additionally, the T2/PD images were used to check for infratentorial WM signal abnormalities because FLAIR imaging is known to be insensitive in this area. The WM signal abnormality number and volume (WM-SAV) were outlined by means of a semi-automated edge detection contouring/thresholding technique as described previously. ${ }^{25}$ All WM signal abnormalities were divided into in- 


\section{Okuda RIS criteria $^{13}$}

1) MRI criteria

a) Ovoid, well-circumscribed, and homogeneous foci with or without involvement of corpus callosum.

b) T2 hyperintensities measuring $>3 \mathrm{~mm}$ in size fulfilling at least 3 of 4 Barkhof criteria ${ }^{14}$ for DIS, which are: 1 ) $\geq 9$ lesions or $\geq 1$ gadolinium-enhancing lesion, 2) $\geq 3$ periventricular lesions, 3) $\geq 1$ juxtacortical lesion, and 4) $\geq 1$ infratentorial lesion.

c) CNS white matter anomalies are not consistent with a vascular pattern.

2) No historical accounts of remitting clinical symptoms consistent with neurologic dysfunction.

3) The MRI anomalies do not account for clinically apparent impairments in social, occupational, or generalized areas of functioning.

4) The MRI anomalies are not due to the direct physiologic effects of substances (eg, drug abuse, toxic exposure) or a medical condition.

5) Exclusion of individuals with MRI phenotypes suggestive of leukoaraiosis or extensive white matter pathology lacking involvement of the corpus callosum.

6) The MRI anomalies are not better accounted for by another disease process.

Swanton RIS criteria $^{17}$

1) MRI criteria

a) DIS defined as presence of $\geq 1$ T2 lesion in $\geq 2$ of the following brain regions (periventricular, juxtacortical, and infratentorial) or in the spinal cord.

2) No historical accounts of remitting clinical symptoms consistent with neurologic dysfunction.

3) The MRI anomalies do not account for clinically apparent impairments in social, occupational, or generalized areas of functioning.

4) The MRI anomalies are not due to the direct physiologic effects of substances (eg, drug abuse, toxic exposure) or a medical condition.

5) Exclusion of individuals with MRI phenotypes suggestive of leukoaraiosis or extensive white matter pathology lacking involvement of the corpus callosum.

6) The MRI anomalies are not better accounted for by another disease process.
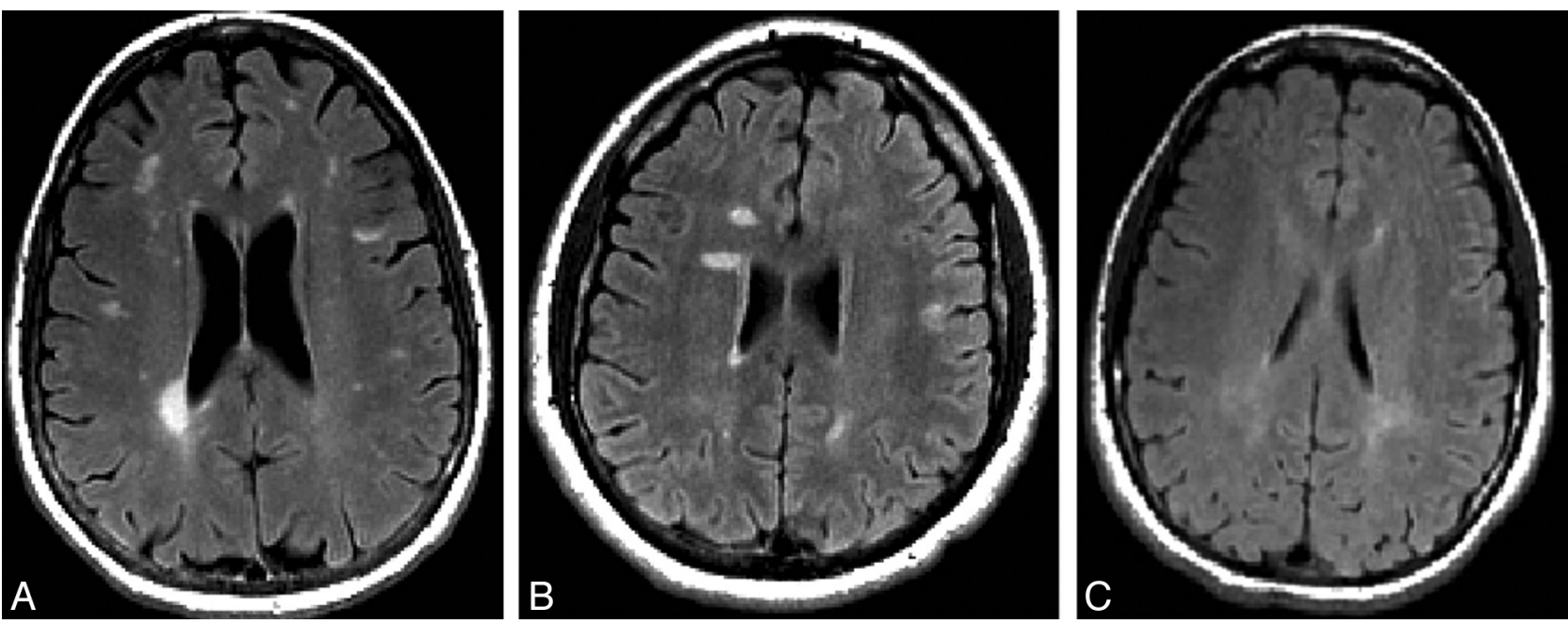

FIG 1. Representative images of the healthy relatives of patients with MS fulfilling Okuda criteria ${ }^{13}$ for radiologically isolated syndrome (RIS) ( $A$ ) fulfilling modified Okuda criteria ${ }^{13}$ for RIS in which the dissemination in space on MR imaging was evaluated by use of Swanton criteria (RIS-Swanton), ${ }^{17}(B)$ or presenting with dirty-appearing white matter (C).

dividual ROIs $\geq 3 \mathrm{~mm}$ in size (equivalent to $\geq 14.1 \mathrm{~mm}^{3}$ ) and ROIs of all sizes (including those $<3 \mathrm{~mm}$ in size). The regional localization of WM signal abnormalities was determined on the basis of their presence in the juxtacortical, periventricular, infratentorial, and deep WM regions.

In addition, we outlined areas of dirty-appearing white matter (DAWM). The DAWM was defined as a uniform, nonfocal area of signal increase on the FLAIR/T2/PD-weighted sequence at 3T, with a subtly increased signal intensity compared with the contralateral signal intensity of normal-appearing WM but less than that of WM signal abnormalities, as previously proposed. ${ }^{26}$ The DAWM measurement was not based on a size, as shown in Fig 1. The DAWM showed a border of areas that were not sharply defined compared with focal WM lesions and was tapered off toward the normal-appearing WM.

\section{Statistical Analysis}

Statistical analyses were performed with the Statistical Package for the Social Sciences, version 16.0 (IBM, Armonk, New York). Demographic, clinical, and MR imaging differences were tested between non-fHCs and the healthy relatives of patients with MS as well as between sporadic and familial HC subgroups. There were no significant differences between sporadic and familial HC subgroups in any of the exploited variables. Therefore, to decrease the number of multiple comparisons, all analyses were performed between non-fHCs and the healthy relatives of patients with MS. For statistics between the groups, the $t$ test, $\chi^{2}$ test, and Mann-Whitney rank sum $U$ test were used as appropriate.

To explore whether there was a relationship between the presence of WM signal abnormalities, $\geq 9$ WM signal abnormalities, 
Table 2: Demographic and clinical characteristics of non-familial healthy control subjects and the healthy relatives of patients with multiple sclerosis

\begin{tabular}{lccc}
\hline & $\begin{array}{c}\text { Non-Familial HCs } \\
(\boldsymbol{n}=82)\end{array}$ & $\begin{array}{c}\text { Healthy Relatives } \\
\text { of Patients with } \\
\text { MS }(\boldsymbol{n}=68)\end{array}$ & $\boldsymbol{P}$ Value \\
\hline Age, y, mean (SD) median & $39.8(14.3) 43$ & $39.5(16.6) 43$ & .917 \\
Sex, female $n(\%)$ & $57(69.5)$ & $45(66.2)$ & .663 \\
Race/ethnicity, $n$ (\%) & & & .180 \\
$\quad$ White & $64(78.1)$ & $62(91.2)$ & \\
Black & $12(14.6)$ & $4(5.9)$ & \\
Other & $6(7.3)$ & $2(2.9)$ & \\
Vascular risk factors, $n(\%)$ & & & .085 \\
Heart disease & $8(9.8)$ & $13(19.1)$ & .979 \\
Smoking & $22(27.5)$ & $19(27.9)$ & .442 \\
Hypertension & $17(20.7)$ & $15(22.1)$ & .582 \\
Obesity $n$ (\%) & & & \\
BMI <18.5 & $2(2.4)$ & $2(2.9)$ & \\
BMI 18.5-24.9 & $34(41.5)$ & $25(36.8)$ & \\
BMI 25-29.9 & $26(31.7)$ & $17(25.0)$ & \\
BMI $>30$ & $13(15.8)$ & $18(26.5)$ & \\
Autoimmune diseases, $n$ (\%) & & & .474 \\
Systemic lupus erythematosus & 0 & 0 & .279 \\
Rheumatoid disorder & $1(1.2)$ & $2(2.9)$ & .352 \\
Psoriasis & 0 & $1(1.5)$ & .282 \\
Diabetes mellitus type 1 & $1(1.2)$ & 0 & \\
Migraine, $n$ (\%) & $10(12.2)$ & $13(19.1)$ & \\
\hline
\end{tabular}

Note:-BMI indicates body mass index.

$\mathrm{BMI}<18.5$ represents underweight, BMI 18.5-24.9 represents normal weight, BMI 25-29.9 represents overweight, and BMI $>30$ represents obesity. The differences between the groups were compared by means of the Student $t$ test or the $\chi^{2}$ test. ${ }^{\text {a }}$ Data missing for 13 subjects.

Table 3: MRI white matter signal abnormality ( $\geq 3 \mathrm{~mm}$ in size) characteristics of nonfamilial healthy control subjects and the healthy relatives of patients with multiple sclerosis

\begin{tabular}{|c|c|c|c|}
\hline & $\begin{array}{l}\text { Non-Familial HCs } \\
\qquad(n=82)\end{array}$ & $\begin{array}{c}\text { Healthy Relatives } \\
\text { of Patients With } \\
\text { MS }(n=68)\end{array}$ & $P$ Value \\
\hline Subjects with WM SAs, $n(\%)$ & $21(25.6)$ & $20(29.4)$ & .603 \\
\hline Subjects with WM JC SAs, $n(\%)$ & $4(4.9)$ & $7(10.3)$ & .205 \\
\hline Subjects with WM PVL SAs, $n(\%)$ & $7(8.5)$ & $13(19.1)$ & .058 \\
\hline Subjects with WM IT SAs, $n(\%)$ & $0(0)$ & $1(1.5)$ & .271 \\
\hline Subjects with DWM SAs, $n(\%)$ & $20(24.4)$ & $17(25.0)$ & .931 \\
\hline Subjects with $\geq 9$ WM SAs, $n(\%)$ & $4(4.9)$ & $6(8.8)$ & .335 \\
\hline WM-SAN, mean (SD), median & $1.5(4.7) 0$ & $2.1(5.1) 0$ & .527 \\
\hline WM JC SAN, mean (SD), median & $0.06(0.3) 0$ & $0.19(0.7) 0$ & .198 \\
\hline WM PVL SAN, mean (SD), median & $0.32(1.8) 0$ & $0.40(1.2) 0$ & .061 \\
\hline WM IT SAN mean (SD) median & $0.0(0) 0$ & $0.01(0.1) 0$ & .272 \\
\hline WM DWM SAN, mean (SD), median & $1.1(3.0) 0$ & $1.5(3.9) 0$ & .838 \\
\hline WM-SAV, mean (SD), median & $166.1(892.0)$ & $230.4(736.8)$ & .480 \\
\hline DAWM-SAV, ${ }^{a}$ mean (SD), median & $675.3(554.7)$ & $866.2(669.3)$ & .054 \\
\hline WM-SAV + DAWM-SAV, mean (SD), median & 842.3 (1079.7) & 1096.5 (1026.6) & .024 \\
\hline
\end{tabular}

Note:-SAs indicates signal abnormalities; SAN, signal abnormality number; JC, juxtacortical, PVL, periventricular; IT, infratentorial; DWM, deep white matter; SAV, signal abnormality volume.

Differences between the groups were compared by means of the $\chi^{2}$ test or Mann-Whitney $U$ test.

The SAV is expressed in millimeters cubed $\left(\mathrm{mm}^{3}\right)$.

${ }^{a}$ DAWM calculation was not related to the size of the hyperintensities.

the fulfillment of RIS-Okuda and RIS-Swanton criteria, and the presence of vascular risk factors (hypertension, heart disease, smoking, obesity, and diabetes mellitus type 1) as well as migraine, we used a multivariate logistic regression model in which age and sex were used as covariates and the vascular risk factors were used as independent variables. The dependent variables were the presence of WM signal abnormalities, $\geq 9$ WM signal abnormalities, and the fulfillment of RIS-Okuda as well as RIS-Swanton criteria. The analyses were separately performed for non-fHCs and the healthy relatives of patients with MS. All $P$ values were calculated by means of a 2-tailed test. Given the pilot nature of the study, a $P$ value of $<.05$ was considered statistically significant, and a $P$ value of $<.1$ was considered a trend.

\section{RESULTS}

\section{Subject Characteristics}

A total of 150 subjects divided into the 2 groups according to their familial characteristics (non-fHCs $=82$, healthy relatives of patients with MS $=68$ ) were enrolled in this study.

The demographic and clinical characteristics of the subjects are listed in Table 2 . There were no age, sex, or race differences between the study groups. There were also no significant differences between the groups in respect to the presence of vascular risk factors, autoimmune diseases, or migraine.

\section{WM Signal Abnormality Characteristics of the Groups}

MR imaging characteristics of WM signal abnormalities and their regional localization are presented in Tables 3 and 4. WM signal abnormalities were divided into 2 groups: those with size $\geq 3$ $\mathrm{mm}$ (Table 3) and those with all sizes (Table 4). There was significantly higher WM-SAV + DAWM-SAV in the healthy relatives of patients with MS as compared with non-fHCs $(1.10 \mathrm{~mL}$ versus $0.84 \mathrm{~mL}, P=.024$ for those $\geq 3 \mathrm{~mm}$ in size, and $1.13 \mathrm{~mL}$ versus $0.87 \mathrm{~mL}, P=$ .025 for all sizes). A trend was observed in the healthy relatives of patients with MS group compared with non-fHCs for DAWM-SAV $\left(866.2 \mathrm{~mm}^{3}\right.$ versus 675.3 $\mathrm{mm}^{3}, P=.054$ for signal abnormality $>3 \mathrm{~mm}$ and all lesion size groups).

In all lesion size groups, more healthy relatives of patients with MS presented with WM signal abnormalities located in the periventricular WM (20.6\% versus $8.5 \%, P=.034)$ and their number was higher $(0.57$ versus 0.41 , $P=0.43$ ) compared with non-fHCs. In the $\geq 3 \mathrm{~mm}$ lesion group, a trend was observed in the healthy relatives of patients with MS for the periventricular signal abnormality localization and number compared with non-fHC (19.1\% versus $8.5 \%, P=.058$ and 0.40 versus $0.32, P=.061$, respectively).

In total, $29.4 \%$ of the healthy relatives of patients with MS showed WM signal abnormalities versus $25.6 \%$ in non-fHCs for signal abnormality $\geq 3 \mathrm{~mm}$ in size (Table 3 ). The corresponding percentages 
of WM signal abnormalities of all sizes were $41.2 \%$ in the healthy relatives of patients with MS group and $35.4 \%$ in the non-fHC group.

\section{Classification of Subjects According to Criteria for DIS and RIS}

Six $(8.8 \%)$ subjects in the healthy relatives of patients with MS group and $4(4.9 \%)$ in non-fHCs showed $\geq 9$ WM signal abnormalities ( $\geq 3 \mathrm{~mm}$ size, Table 3 ). Of these, all subjects had lesions in 2 location categories in the healthy relatives of patients with MS group, whereas there were 3 of 4 in non-fHCs. The percentages of $\geq 9 \mathrm{WM}$ signal abnormality of all sizes were $11.8 \%$ and $6.1 \%$ in the healthy relatives of patients with MS group and the non-fHC group, respectively. These differences were not significant.

Two $(2.9 \%)$ subjects in the healthy relatives of patients with MS group and $2(2.4 \%)$ non-fHCs fulfilled RIS-Okuda criteria (Table 5). Ten subjects fulfilled the RIS-Swanton: 7 (10.3\%) in the

\begin{tabular}{|c|c|c|c|}
\hline & $\begin{array}{l}\text { Non-Familial HCs } \\
\quad(n=82)\end{array}$ & $\begin{array}{l}\text { Healthy Relatives } \\
\text { of Patients with } \\
\text { MS ( } n=68)\end{array}$ & $P$ Value \\
\hline Subjects with WM SAs, $n(\%)$ & $29(35.4)$ & $28(41.2)$ & .465 \\
\hline Subjects with WM JC SAs, $n(\%)$ & $4(4.9)$ & $7(10.3)$ & .205 \\
\hline Subjects with WM PVL SAs, $n(\%)$ & $7(8.5)$ & $14(20.6)$ & .034 \\
\hline Subjects with WM IT SAs, $n(\%)$ & $1(1.2)$ & $1(1.5) 0$ & .894 \\
\hline Subjects with DWM SAs, $n(\%)$ & $28(34.1)$ & $26(38.2)$ & .603 \\
\hline Subjects with $\geq 9$ WM SAs, $n(\%)$ & $5(6.1)$ & $8(11.8)$ & .219 \\
\hline WM-SAN, mean (SD) median & $2.5(7.5) 0$ & $3.8(10) 0$ & .386 \\
\hline WM JC SAN, mean (SD) median & $0.07(0.3) 0$ & $0.19(0.7) 0$ & .207 \\
\hline WM PVL SAN, mean (SD) median & $0.41(2.1) 0$ & $0.57(1.9) 0$ & .043 \\
\hline WM IT SAN, mean (SD) median & $0.01(0.1) 0$ & $0.01(0.12) 0$ & .894 \\
\hline WM DWM SAN, mean (SD) median & $1.9(5.3) 0$ & $3.1(8.5) 0$ & .468 \\
\hline WM-SAV, mean (SD) median & $198.2(1085.2)$ & $259.7(784.4)$ & .338 \\
\hline DAWM-SAV, ${ }^{a}$ mean (SD) median & $675.3(554.7)$ & $866.2(669.3)$ & .054 \\
\hline WM-SAV + DAWM-SAV, mean (SD) median & $873.5(1233.5)$ & $1125.9(1076.8)$ & .025 \\
\hline
\end{tabular}

Note:-SAs indicates signal abnormalities; SAN, signal abnormality number; JC, juxtacortical; PVL, periventricular; IT, infratentorial; DWM, deep white matter; SAV, signal abnormality volume.

Differences between the groups were compared by means of the $\chi^{2}$ test or Mann-Whitney $U$ test.

The SAV is expressed in millimeters cubed $\left(\mathrm{mm}^{3}\right)$.

${ }^{a}$ DAWM calculation was not related to the size of the hyperintensities. healthy relatives of patients with MS and 3 (3.7\%) non-fHCs. These differences were not significant. Localization characteristics of WM signal abnormalities are listed in Table 5.

\section{Relationship Between Vascular Risk Factors and RIS and WM Signal Abnormality Findings}

In multivariate regression analysis in which the presence of WM signal abnormalities was used as a dependent variable, smoking ( $\mathrm{B}=0.44, P=.024)$ was the only variable associated with the healthy relatives of patients with MS.

In multivariate regression analysis in which $\geq 9$ WM signal abnormalities was used as a dependent variable, obesity (body mass index $>30$ ) was the only variable associated with the healthy relatives of patients with MS ( $\mathrm{B}=0.56, P=.003)$.

In multivariate regression analysis in which RIS-Swanton was used as a dependent variable, obesity $(\mathrm{B}=0.29, P=.019)$ was the only variable associated with the healthy relatives of patients with MS. No association was found in non-fHCs for all 3 dependent variables.

No association between vascular risk factor variables and RIS-Okuda criteria was found in any HC group.

\section{DISCUSSION}

Subjects with WM signal abnormalities suggestive for MS but without history of MS symptoms or neurologic deficit may be at an increased risk for development of the disease. This pilot study included a large cohort of $150 \mathrm{HCs}$ with or without familial history of MS that were collected prospectively by use of 3T MR imaging. We assessed the prevalence of WM signal abnormalities and of RIS in the study groups, also applying the Swanton MR

Table 5: Non-familial healthy control subjects and the healthy relatives of patients with multiple sclerosis fulfilling Okuda criteria for RIS or fulfilling modified Okuda criteria for RIS in which the dissemination in space on MRI was evaluated by use of Swanton criteria (RIS-Swanton)

\begin{tabular}{|c|c|c|c|}
\hline & Non-Familial HCs ( $n=82$ ) & $\begin{array}{c}\text { Healthy Relatives } \\
\text { of Patients with MS }(n=68)\end{array}$ & $P$ Value \\
\hline \multicolumn{4}{|c|}{ RIS-Okuda criteria ${ }^{13}$} \\
\hline Non-RIS & 80 & 66 & .849 \\
\hline RIS & 2 & 2 & \\
\hline Subject 1 & >9 WM SA: 5 JC, 3 PVL, 13 DWM & >9 WM SA: 5 JC, 3 PVL, 13 DWM & \\
\hline Subject 2 & >9 WM SA: $1 \mathrm{JC}, 8$ PVL, 11 DWM & >9 WM SA: 1JC, 8 PVL, 11 DWM & \\
\hline Total & 82 & 68 & \\
\hline \multicolumn{4}{|c|}{ Modified Okuda criteria (RIS-Swanton ${ }^{13,17}$ ) } \\
\hline Non-RIS & 79 & 61 & .105 \\
\hline RIS & 3 & 7 & \\
\hline Subject 1 & $>9$ WM SA: 2 JC, 5 PVL, 9 DWM & >9 WM SA: $5 \mathrm{JC}, 3$ PVL, 13 DWM & \\
\hline Subject 2 & >9 WM SA: $1 \mathrm{JC}, 15$ PVL, 18 DWM & >9 WM SA: 1JC, 8 PVL, 11 DWM & \\
\hline Subject 3 & >9 WM SA: $1 \mathrm{JC}, 1 \mathrm{PVL}, 11 \mathrm{DWM}$ & $>9$ WM SA: 2 JC, 1 PVL, 7 DWM & \\
\hline Subject 4 & & >9 WM SA: $1 \mathrm{JC}, 1 \mathrm{PVL}, 24 \mathrm{DWM}$ & \\
\hline Subject 5 & & >9 WM SA: 1JC, 1 PVL, 7 DWM & \\
\hline Subject 6 & & >9 WM SA: 1JC, 1 PVL, 8 DWM & \\
\hline Subject 7 & & $2 \mathrm{JC}, 2$ PVL 3 DWM & \\
\hline Total & 82 & 68 & \\
\hline
\end{tabular}

Note:-SA indicates signal abnormality; JC, juxtacortical; PVL, periventricular; DWM, deep white matter.

Differences between the groups were compared by means of the $\chi^{2}$ test. The regional localization data for subjects fulfilling RIS criteria are presented on individual subject level. 
imaging criteria for DIS. ${ }^{17}$ There was a significantly higher total volume of WM signal abnormalities (including WM-SAV + DAWMSAV) in subjects with familial MS history. In these subjects, significantly more WM signal abnormalities (only for all WM signal abnormality sizes) were located in periventricular WM compared with non-fHCs. No significant differences were observed between the groups for presence, number, or localization of juxtacortical, infratentorial, and deep WM signal abnormalities. Although more subjects in the group with familial history of MS presented with RIS according to Okuda et $\mathrm{al}^{13}$ or modified Okuda criteria (in which DIS was evaluated according to the Swanton MR imaging criteria), the differences were not significant. Interestingly, in the healthy relatives of MS patients in multivariate regression models, smoking was associated with the presence of WM signal abnormalities, while obesity was related to the presence of $\geq 9 \mathrm{WM}$ signal abnormalities and fulfillment of RIS-Swanton criteria. This is in line with recently reported data in pediatric patients with $\mathrm{MS}^{27}$

Increasing diagnostic application of MR imaging, especially at higher strength field, results in an increased number of abnormal incidental findings with potential clinical significance. ${ }^{28,29} \mathrm{Sev}-$ eral postmortem and MR imaging studies aimed to establish the prevalence of demyelinating lesions, possibly indicative of RIS. ${ }^{28-31}$ Disadvantages of postmortem studies are related to the fact that they are usually performed at late stages of the disease (autopsies) or in selected atypical early cases (biopsies), which analyze the brain only in 1 anatomic part. In comparison with $\mathrm{MR}$ imaging, pathologic assessment does not allow observation of the evolution of pathologic changes over time. A recent MR imaging meta-analysis included 15,559 healthy adults and found only 9 cases of definite demyelination $(0.06 \%){ }^{28}$ This meta-analysis included studies performed on MR imaging magnets with field strengths $1 \mathrm{~T}$ or $1.5 \mathrm{~T}$. The most common reason for performing MR imaging among patients later identified as having RIS is headache, ${ }^{15}$ which is also described as a presenting MS symptom in some cases. ${ }^{32}$ Compared with these previous studies, the present one evaluated asymptomatic HCs with no signs or symptoms of MS. Despite this, the prevalence of RIS on the basis of RIS-Okuda or RIS-Swanton criteria was higher in subjects with $(2.9 \%$ and $10.3 \%$, respectively) compared with those without $(2.4 \%$ and $3.7 \%$, respectively) family MS history.

Although the incidence of MS among relatives is increased in affected families, ${ }^{1}$ there are only a few MR imaging studies that evaluated the WM signal abnormalities of asymptomatic healthy relatives of patients with MS. ${ }^{20-22}$ All were performed on 1T or 1.5T MR imaging. In the current study, we used 3T MR imaging, which can substantially increase the detection of WM and DAWM abnormalities. ${ }^{33,34}$ A previous $3 \mathrm{~T}$ versus $1.5 \mathrm{~T}$ comparison study in patients with MS and HCs showed regional predilection of WM signal abnormality detection in periventricular, pericallosal, cortical/juxtracortical, and infratentorial regions on 3T MR imaging. ${ }^{33}$ The correlation of MR imaging findings with clinical status is also stronger at $3 \mathrm{~T}$ compared with $1.5 \mathrm{~T}$ MR imaging. ${ }^{35}$ The use of $3 \mathrm{~T}$ may be one of the reasons why we found a significantly higher total volume of WM signal abnormalities (including WM-SAV + DAWM-SAV) in patients with familial MS history as compared with those without history. Regional predilection and number of WM signal abnormalities was detected only for the periventricular area in the healthy relatives of patients with MS. No regional predilection of WM signal abnormalities has been detected between the study groups for both the presence and number of other anatomic sites. Previous studies in asymptomatic healthy relatives of patients with MS did not report WM signal abnormality number or size. ${ }^{20-22}$ Our study showed no significant differences between the $\mathrm{HC}$ groups in the presence or number of WM signal abnormalities $\geq 3 \mathrm{~mm}$ in size or when all WM signal abnormalities, independent of the size, were added to the total count.

WM signal abnormalities on MR imaging are more frequent in older individuals and in subjects presenting with vascular risk factors $^{36,37}$; therefore their presence in asymptomatic individuals should be interpreted with caution. No differences between the HC with or without familial MS history were found for demographic characteristics, vascular risk factors, migraine, or the presence of autoimmune diseases considered in this study. However, in regression analysis, we found that only the relatives of subjects with MS who showed WM signal abnormalities or had $\geq 9$ WM signal abnormalities and fulfilled RIS-Swanton criteria had elevated smoking and obesity vascular risk factors. It could be hypothesized that these risk factors may play a different role in formation of WM signal abnormalities in individuals genetically susceptible for MS, as are relatives of patients with MS. A recent study suggested that primary lipid abnormality in DAWM may be involved in demyelination and axonal degeneration of patients with $\mathrm{MS},{ }^{38}$ and obesity was recently suggested as linked with increased morbidity for MS. ${ }^{27}$

Persons with RIS are at an increased risk of development of MS. ${ }^{39,40}$ During a follow-up period of $2-5$ years, approximately one-third of patients had clinical symptoms characteristic of MS. ${ }^{39,40}$ Increased risk for clinical conversion was observed in younger subjects, with a higher number of lesions, presence of infratentorial lesions, pathologic evoked potentials, positive oligoclonal bands, and spinal cord lesions. ${ }^{40}$ The limitation of our study was that it was a cross-sectional pilot study that did not include spinal cord MR imaging, and no information is currently available about longitudinal follow-up of the enrolled subjects.

This is the first study in which the Swanton MR imaging criteria for DIS ${ }^{17}$ were integrated in the Okuda criteria for RIS. ${ }^{13}$ The prevalence of RIS-Swanton in the healthy relatives of patients with MS was increased compared with those without, whereas no differences were found when RIS-Okuda criteria ${ }^{13}$ were applied. Future longitudinal studies should determine whether asymptomatic HCs fulfilling RIS-Swanton criteria have an increased risk of development of MS as compared with RIS-Okuda criteria. ${ }^{13}$ De Stefano et $\mathrm{al}^{20}$ reported that $7.1 \%$ of the relatives of patients with MS fulfilled Barkhof criteria $^{14}$ on MR imaging for DIS. Although $8.8 \%$ of the healthy relatives of patients with MS showed $\geq 9$ WM signal abnormalities, only $2.9 \%$ presented with RIS-Okuda. However, $2.4 \%$ of HCs without MS family history also fulfilled RIS-Okuda criteria in the present study, compared with none fulfilling Barkhof criteria for DIS ${ }^{14}$ in the De Stefano et $\mathrm{al}^{20}$ study. The reasons for these differences are not clear and should be further explored.

To establish the long-term value of RIS Okuda and Swanton criteria on clinical and imaging follow-up of our study cohort, a 5 -year follow-up is planned. Because the treatment of RIS is controversial, possible differences in the conversion rate of clinical and 
imaging characteristics between the RIS Okuda/Swanton criteria may influence future treatment decisions at the stage of RIS.

\section{CONCLUSIONS}

The healthy relatives of patients with MS show more frequent WM signal abnormalities as compared with those without and are more likely to present with RIS when Swanton criteria for DIS are applied.

ICMJE Disclosures: Robert Zivadinov—UNRELATED: Consultancy: Biogen Idec, EMD Serono, Teva Pharmaceuticals, Genzyme-Sanof, Novartis; Grants/Grants Pending: Biogen Idec, ${ }^{\star}$ EMD Serono, ${ }^{*}$ Teva Pharmaceuticals, ${ }^{*}$ Novartis, ${ }^{*}$ Genzyme-Sanofi*; Payment for Lectures (including service on speaker's bureaus): Biogen Idec, EMD Serono, Teva Pharmaceuticals, Genzyme-Sanofi, Novartis. Bianca Weinstock-Guttmann—UNRELATED: Consultancy: Biogen Idec, Teva Neurosciences, EMD Serono, Novartis, Genzyme, Acorda; Grants/Grants Pending; Payment for Lectures (including service on speakers bureaus): Teva Neuroscience, Biogen Idec, EMD Serono, Novartis, Pfizer, Genzyme, and Acorda. David Hojnacki—UNRELATED: Consultancy: Teva, Biogen, EMD, Pfizer; Payment for Lectures (including service on speaker's bureaus): Teva, Biogen, EMD, Pfizer. Murali Ramanathan-UNRELATED: Grants/Grants Pending: National Multiple Sclerosis Society, ${ }^{\star}$ Department of Defense*; Other: Self-published textbook on Pharmacy Calculations ( ${ }^{*}$ money paid to institution).

\section{REFERENCES}

1. Compston A, Coles A. Multiple sclerosis. Lancet 2002;359:1221-31

2. Weinshenker BG, Bulman D, Carriere W, et al. A comparison of sporadic and familial multiple sclerosis. Neurology 1990;40:1354-58

3. Siger-Zajdel M, Filippi M, Selmaj K. MTR discloses subtle changes in the normal-appearing tissue from relatives of patients with MS. Neurology 2002;58:317-20

4. O'Gorman C, Lin R, Stankovich J, et al. Modelling genetic susceptibility to multiple sclerosis with family data. Neuroepidemiology 2013;40:1-12

5. Sadovnick AD, Yee IM, Ebers GC, et al. Effect of age at onset and parental disease status on sibling risks for MS. Neurology 1998;50:719-23

6. Prokopenko I, Montomoli C, Ferrai R, et al. Risk for relatives of patients with multiple sclerosis in central Sardinia, Italy. Neuroepidemiology 2003;22:290-96

7. Ramagopalan SV, Dobson R, Meier UC, et al. Multiple sclerosis: risk factors, prodromes, and potential causal pathways. Lancet Neurol 2010;9:727-39

8. Hensiek AE, Seaman SR, Barcellos LF, et al. Familial effects on the clinical course of multiple sclerosis. Neurology 2007;68:376-83

9. Ebers GC, Koopman WJ, Hader W, et al. The natural history of multiple sclerosis: a geographically based study: 8: familial multiple sclerosis. Brain 2000;123(Part 3):641-49

10. Tipirneni A, Weinstock-Guttman B, Ramanathan M, et al. MRI characteristics of familial and sporadic multiple sclerosis patients. Mult Scler Dec 11, 2012 [Epub ahead of print]

11. Siger-Zajdel M, Selmaj K. Magnetisation transfer ratio analysis of normal appearing white matter in patients with familial and sporadic multiple sclerosis. J Neurol Neurosurg Psychiatry 2001;71:752-56

12. Siger-Zajdel M, Selmaj KW. Proton magnetic resonance spectroscopy of normal appearing white matter in familial and sporadic multiple sclerosis. J Neurol 2005;252:830-32

13. Okuda DT, Mowry EM, Beheshtian A, et al. Incidental MRI anomalies suggestive of multiple sclerosis: the radiologically isolated syndrome. Neurology 2009;72:800-05

14. Barkhof F, Filippi M, Miller DH, et al. Comparison of MRI criteria at first presentation to predict conversion to clinically definite multiple sclerosis. Brain 1997;120(Part 11):2059-69

15. Granberg T, Martola J, Kristoffersen-Wiberg M, et al. Radiologically isolated syndrome: incidental magnetic resonance imaging findings suggestive of multiple sclerosis, a systematic review. Mult Scler 2013;19:271-80

16. Polman CH, Reingold SC, Banwell B, et al. Diagnostic criteria for multiple sclerosis: 2010 revisions to the McDonald criteria. Ann Neurol 2011;69:292-302

17. Swanton JK, Fernando K, Dalton CM, et al. Modification of MRI criteria for multiple sclerosis in patients with clinically isolated syndromes. J Neurol Neurosurg Psychiatry 2006;77:830-33

18. De Stefano N, Stromillo ML, Rossi F, et al. Improving the characterization of radiologically isolated syndrome suggestive of multiple sclerosis. PLoS One 2011;6:e19452

19. Filippi M, Campi A, Martino G, et al. A magnetization transfer study of white matter in siblings of multiple sclerosis patients. J Neurol Sci 1997;147:151-53

20. De Stefano N, Cocco E, Lai M, et al. Imaging brain damage in firstdegree relatives of sporadic and familial multiple sclerosis. Ann Neurol 2006;59:634-39

21. Tienari PJ, Salonen O, Wikstrom J, et al. Familial multiple sclerosis: MRI findings in clinically affected and unaffected siblings. J Neurol Neurosurg Psychiatry 1992;55:883-86

22. Lynch SG, Rose JW, Smoker W, et al. MRI in familial multiple sclerosis. Neurology 1990;40:900-03

23. Dolic K, Weinstock-Guttman B, Marr K, et al. Risk factors for chronic cerebrospinal venous insufficiency (CCSVI) in a large cohort of volunteers. PLoS One 2011;6:e28062

24. Jenkinson M, Bannister P, Brady M, et al. Improved optimization for the robust and accurate linear registration and motion correction of brain images. Neuroimage 2002;17:825-41

25. Zivadinov R, Rudick RA, De Masi R, et al. Effects of IV methylprednisolone on brain atrophy in relapsing-remitting MS. Neurology 2001;57:1239-47

26. Seewann A, Vrenken H, van der Valk P, et al. Diffusely abnormal white matter in chronic multiple sclerosis: imaging and histopathologic analysis. Arch Neurol 2009;66:601-09

27. Langer-Gould A, Brara S, Beaber B, et al. Childhood obesity and risk of pediatric multiple sclerosis and clinically isolated syndrome. Neurology 2013;80:548-52

28. Morris Z, Whiteley WN, Longstreth WT Jr, et al. Incidental findings on brain magnetic resonance imaging: systematic review and metaanalysis. BMJ 2009;339:b3016

29. Weber F, Knopf H. Incidental findings in magnetic resonance imaging of the brains of healthy young men. J Neurol Sci 2006;240:81-84

30. Engell T. A clinical patho-anatomical study of clinically silent multiple sclerosis. Acta Neurol Scand 1989;79:428-30

31. Wasay M, Rizvi F, Azeemuddin M, et al. Incidental MRI lesions suggestive of multiple sclerosis in asymptomatic patients in Karachi, Pakistan. J Neurol Neurosurg Psychiatry 2011;82:83-85

32. D'Amico D, La Mantia L, Rigamonti A, et al. Prevalence of primary headaches in people with multiple sclerosis. Cephalalgia 2004;24:980-84

33. Di Perri C, Dwyer MG, Wack DS, et al. Signal abnormalities on 1.5 and 3 Tesla brain MRI in multiple sclerosis patients and healthy controls: a morphological and spatial quantitative comparison study. Neuroimage 2009;47:1352-62

34. Sicotte NL, Voskuhl RR, Bouvier S, et al. Comparison of multiple sclerosis lesions at 1.5 and 3.0 Tesla. Invest Radiol 2003;38:423-27

35. Stankiewicz JM, Glanz BI, Healy BC, et al. Brain MRI lesion load at $1.5 \mathrm{~T}$ and $3 \mathrm{~T}$ versus clinical status in multiple sclerosis. J Neuroimaging 2011;21:e50-56

36. Enzinger C, Smith S, Fazekas F, et al. Lesion probability maps of white matter hyperintensities in elderly individuals: results of the Austrian stroke prevention study. J Neurol 2006;253:1064-70

37. Gouw AA, Seewann A, van der Flier WM, et al. Heterogeneity of small vessel disease: a systematic review of MRI and histopathology correlations. J Neurol Neurosurg Psychiatry 2011;82:126-35

38. Laule C, Pavlova V, Leung E, et al. Diffusely abnormal white matter in multiple sclerosis: further histologic studies provide evidence for a primary lipid abnormality with neurodegeneration. J Neuropathol Exp Neurol 2013;72:42-52

39. Lebrun C, Bensa C, Debouverie M, et al. Unexpected multiple sclerosis: follow-up of 30 patients with magnetic resonance imaging and clinical conversion profile. J Neurol Neurosurg Psychiatry 2008;79:195-98

40. Okuda DT, Mowry EM, Cree BA, et al. Asymptomatic spinal cord lesions predict disease progression in radiologically isolated syndrome. Neurology 2011;76:686-92 